\title{
Relativistic tidal disruption events
}

\author{
A. Levan ${ }^{a}$ for a larger collaboration \\ Department of Physics, University of Warwick, Coventry, CV4 7AL, UK
}

\begin{abstract}
In March 2011 Swift detected an extremely luminous and long-lived outburst from the nucleus of an otherwise quiescent, low luminosity (LMC-like) galaxy. Named Swift J1644+57, its combination of high-energy luminosity $\left(10^{48} \mathrm{ergs} \mathrm{s}^{-1}\right.$ at peak), rapid X-ray variability (factors of $>100$ on timescales of 100 seconds) and luminous, rising radio emission suggested that we were witnessing the birth of a moderately relativistic jet $(\Gamma \sim 2-5)$, created when a star is tidally disrupted by the supermassive black hole in the centre of the galaxy. A second event, Swift J2058+0516, detected two months later, with broadly similar properties lends further weight to this interpretation. Taken together this suggests that a fraction of tidal disruption events do indeed create relativistic outflows, demonstrates their detectability, and also implies that low mass galaxies can host massive black holes. Here, I briefly outline the observational properties of these relativistic tidal flares observed last year, and their evolution over the first year since their discovery.
\end{abstract}

\section{INTRODUCTION}

On the 28th March 2011 the Swift satellite detected what appeared at first to be a very long duration gamma-ray burst (GRB 110328A), detected as an image trigger with a duration of over twenty minutes [1]. A prompt slew after this detection revealed a bright X-ray source, which rather than decaying as GRB afterglows do, subsequently brightened, triggering the gamma-ray detectors of the Burst Alert Telescope (BAT) again roughly 40 minutes later [2] - something almost unheard of for GRBs.

An analysis of archival images of the field revealed a quiescent counterpart with $\mathrm{R} \sim 22$ in the $\mathrm{X}$-ray error box [3]. Combined with the luminous X-ray afterglow these properties appeared at first sight similar to those of the Supergiant Fast X-ray Transients - High mass X-ray binaries with our Galaxy [4]. However, our spectra of the source obtained the following night in fact showed it to be a star-forming galaxy at $z=0.35$ [5], and immediately implied an extremely unusual extragalactic transient, whose luminosity and longevity were unlike any object previously seen. Our observations also unveiled a faint, fading infrared counterpart [6], which would ultimately provide a precise position for the burst relative to this host galaxy [7].

The X-ray afterglow was seen to persist at a bright level $\left(>10^{-10} \operatorname{ergs~} \mathrm{s}^{-1} \mathrm{~cm}^{-2}\right)$ for several days, and indeed subsequently re-triggered the BAT on two further occasions (i.e. triggered it four times in total) [2]. These properties defied explanation in standard GRB paradigms in which the progenitor star explodes as a $\mathrm{SN}$, and the power to the newly formed stellar mass black hole engine decreases extremely rapidly. However, it did appear to fit with the timescales associated with tidal disruption events (TDEs). In this case the challenge was to explain the extreme luminosity, which at peak was over $10^{48}$ ergs $\mathrm{s}^{-1}$. However, TDEs in which a relativistic component is also launched had be proposed [8,9], and so it was suggested by Bloom et al. [10], that in GRB 110328A (subsequently renamed, and more commonly known as

a e-mail: a.j.levan@warwick.ac.uk
Swift J1644+57), that we were actually observing a relativistic tidal disruption event which was behaving as a "mini-blazar", a suggestion that has continued to be supported by further observations [11-13].

Interestingly, despite the unprecedented properties of Swift 1644+57, a second event, which looks remarkably similar was discovered only two months later. Named Swift $\mathrm{J} 2058+0516$, it also exhibits luminous long-lived X-ray emission, accompanied by a fading optical/IR source, but at a distance of $z=1.18$ [18].

Here I briefly describe the observational properties of Swift J1644+57 and Swift J2058+0516, and how they have evolved over the first year since their discovery.

\section{SWIFT J1644+57}

Observations of Swift $\mathrm{J} 1644+57$ are described in detail in numerous papers [7,11-16]. To Briefly summarize, it exhibits an extremely luminous X-ray and $\gamma$-ray counterpart, which has was detected at luminous flux levels, with a peak of $\sim 3 \times 10^{48} \mathrm{ergs} \mathrm{s}^{-1}$, and persisted at $>10^{45} \mathrm{ergs} \mathrm{s}^{-1}$ for in excess of a year. This is punctuated throughout by significant variability (in particular dips of a factor of ten or more), although this is most pronounced at early times, during which the flux is seen to change by factors of up to 600 in only a few hundred seconds. The peak luminosity exceeds the Eddington limit for a $10^{10} \mathrm{M}_{\odot}$ black hole. For the majority of the first year since the outburst, the over decay rate of the X-ray luminosity (omitting the "dips") was remarkably close to the canonical $t^{-5 / 3}$ expected from the fallback of disrupted material into the disc (see Figure 1). Interestingly, having persisted at such luminous levels for in excess of year, Swift monitoring of Swift J1644+57 has indicated a rapid drop in luminosity in September and October 2012 [17]. This may be indicative of the relativistic jet finally switching off.

There is no evidence of optical variability within the host to deep limits $(R>25)$ based on extensive Gemini observations. However, at IR wavelengths (essentially zband and redward) transient emission is seen, which gets 


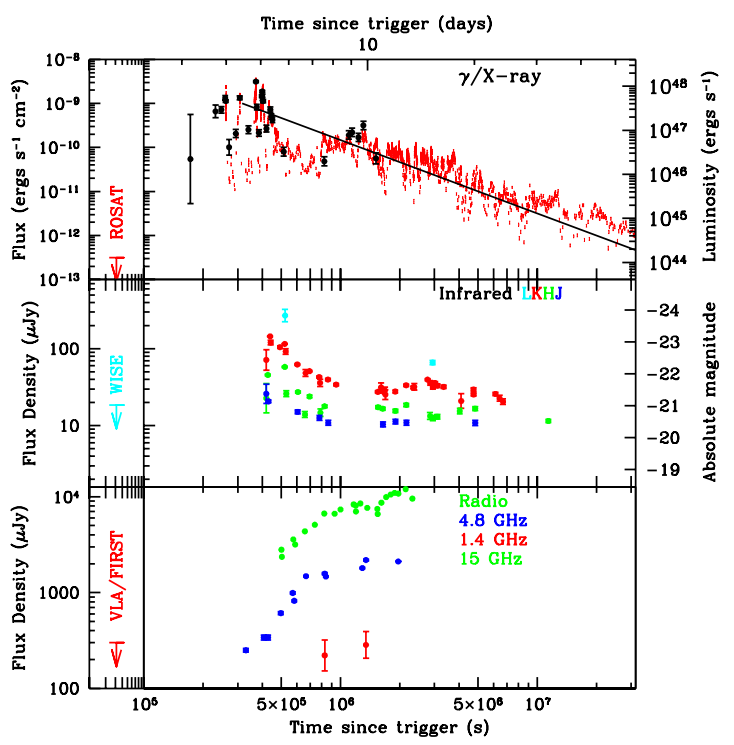

Figure 1. Multiwavelength $\gamma / \mathrm{X}$-ray, optical/IR and radio light curves of Swift J1644+57, adapted from [7]. The panels show increasing wavelength, from $\gamma / \mathrm{X}$-ray (top), optical/IR (middle) to radio (lower). The lightcurves clearly vary between these wavelengths, but are extremely luminous in all of them. Of particular note is i) the rapid variability implied in the X-ray lightcurves, ii) the very red colour seen in the optical/IR (data from $[7,12]$ and supplemented with more recent observations) and iii) the late time rise seen in the radio, indicative of significant late time energy input (data from [7,14].

stronger towards longer wavelengths, and is particularly pronounced in the mid-IR (ground based L-band and with Spitzer) [7]. Such a scenario is broadly consistent with extinction of $A_{V} \sim 4$, and also agrees with the high $N_{H}$ inferred from $\mathrm{X}$-ray observations. There is some evidence of re-brightening in the IR on timescales of $\sim 1$ month post burst, perhaps temporally coincident with the peak of the radio flux (see below).

A crucial diagnostic of the nature of Swift J1644+57 comes from the precise positioning of the transient on its host galaxy, by aligning observations in the IR (where the transient dominates) with those in the optical it appears to originate $<150 \mathrm{pc}$ from the nucleus of its host. Although the host is compact (roughly $1 \mathrm{kpc}$ across) this strongly suggests an origin in the supermassive black hole within the nucleus [7].

At radio wavelengths the source is also strongly detected, and exhibits a large scale rise in luminosity, beginning roughly 30 days after the initial outburst [14], that is indicative of significant late time energy input. Utilizing standard blast-wave models the radio counterpart also offers a means of measuring the expanding source size, and implies that the outflow is indeed moderately relativistic, with $\Gamma \sim 2$ [13].

\section{SWIFT J2058+0516}

Observations of Swift J2058+0516 are described in detail in Cenko et al. [18]. It was discovered by the Swift BAT transient monitor, rather than as a GRB trigger, but appears

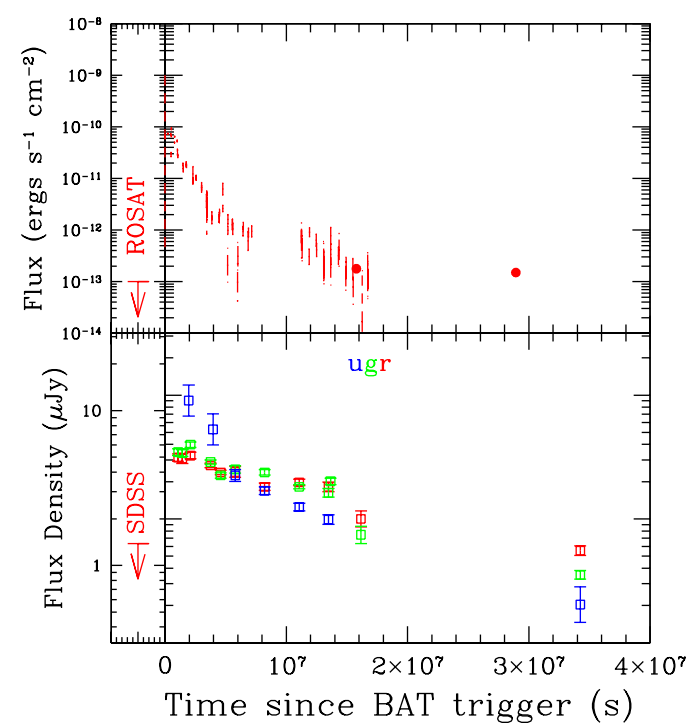

Figure 2. The X-ray (top) and UV/optical (bottom) light curves of Swift J2058+0516, spanning the first year since the discovery of the outburst. The optical decay is seen to be relatively slow at first, while the X-ray decays more rapidly. At later times the $\mathrm{X}$-ray appears to flatten as indicated by the approximate position of two $X M M$-Newton observations of the source (solid red dots). The optical light declines quickly after 100 days, and may be dominated by a host galaxy at late times, although the object remains point-like.

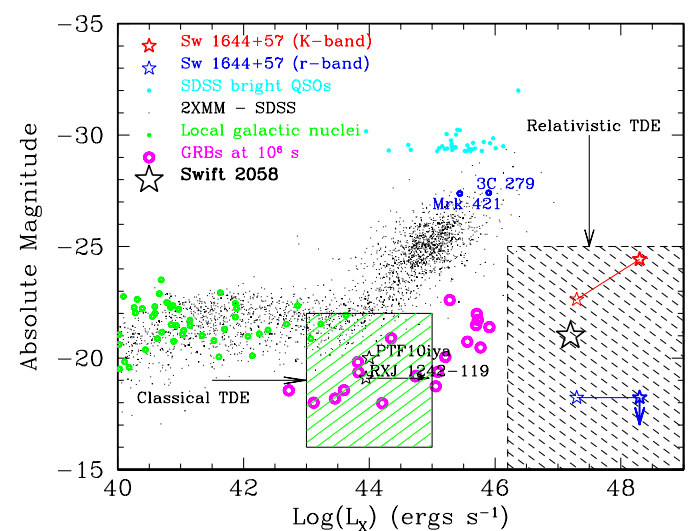

Figure 3. The location of various luminous sources in the $X$ ray luminosity versus absolute magnitude plane, with the type of event indicated by the inset key. This includes standard active galaxies, luminous quasars and blazars, and normal lower luminosity galactic nuclei in the local Universe. Also shown are the locations of various transient sources, including gammaray bursts at late times after outburst, and candidate nonrelativistic TDEs (green shaded area). The relativistic TDEs are much more X-ray luminous (while comparably optically faint) than other sources, this may make their identification relatively straightforward with snapshot observations.

as a luminous $\gamma$ - and X-ray transient, which lasts for several days (it is visible in a 4-day integration with the BAT). It was accompanied by a moderately bright optical counterpart, which unlike Swift J1644+57 was extremely blue, indicative of very little extinction. A spectrum obtained by Keck showed a redshift of $z=1.18$, based on the presence of Fe absorption lines, again marking 
Swift $\mathbf{J} 2058+0516$ as a luminous extragalactic flare, with a peak luminosity in excess of $10^{48} \mathrm{ergs} \mathrm{s}^{-1}$ [18]. While the distance to Swift J2058+0516 make it more challenging to study, the lack of extinction provides a much better opportunity to investigate the evolution of its optical counterpart, which in the case of Swift J1644+57 was highly obscured. This counterpart is seen to fade slowly at first, especially in the redder bands, but breaks $\sim 100$ days after the initial identification of the outburst to a steeper decay. In other words, the evolution of the source is clearly achromatic, with a strong blue to red evolution.

The X-ray lightcurve is notable because it also exhibits a degree of rapid variation at early times, and appears to decay rather more rapidly than the canonical $t^{-5 / 3}$, being best fit with a decay of around $t^{-2.2}$. However, late time XMM-Newton observations appear to show that this decay plateau's, and it remains to be seen if there is an underlying $\mathrm{X}$-ray source, or if the decay will continue at a later time.

At late times a faint, point-like source is visible at the location of the transient, there is no astrometrically significant offset between this source and the transient itself, so we cannot assess if it is time late time light from the transient itself, or an underlying (but extremely compact) host galaxy. Aside from the spectral absorption lines in the early spectrum there is so far no sign of the host galaxy (Levan et al. in prep).

\section{INTERPRETATION AND IMPICATIONS}

The luminosity of the host galaxy of Swift J1644+57 is $M_{K}(A B) \lesssim-19.5$ [7], while for Swift J2058+0516 it appears to be $M_{R}(A B) \lesssim-20$ ( [18], Levan et al. in prep). Based on the bulge mass - black hole mass relations this suggests that they should not host black holes more massive than $\sim 10^{7} \mathrm{M}_{\odot}$. In this case, the observed X-ray emission at early times is super-Eddington by a factor of a thousand or more (although this may be mitigated somewhat by beaming), and persists at this level for several months. The most straightforward means of resolving this problem is that the accretion flow may have been modestly super-Eddington at early times, but has its total energy significantly altered by relativistic beaming. This is supported by radio observations, where the application of blast wave models allows an inference of the size of the emitting region as a function of time, and suggests that the outflow is moderately relativistic [13].

If correctly interpreted the discovery of these events has several important implications. Firstly, the host galaxies are small, compact and low luminosity. This suggests they harbour low mass black holes, and offers confirmation of the existence of black holes in these low mass galaxies. Secondly, it offers the possibility of searching for TDEs in a potentially much greater volume than previously possible, since the X-ray and radio emission from Swift $1644+57$ would be visible to current generation technology out to $z \sim 6$ [13]. Finally, these events offer confirmation that the tidal disruption of stars can create relativistic emission, and subsequently may be ideal locations for particle acceleration. Given the long-standing problem of accelerating Ultra High Energy Cosmic Rays (UHECRs), these events may provide a natural remedy $[11,18]$.

\section{References}

[1] Cummings, J. R., Barthelmy, S. D., Beardmore, A. P., et al. 2011, GRB Coordinates Network, 11823, 1

[2] Sakamoto, T., Barthelmy, S. D., Baumgartner, W. H., et al. 2011, GRB Coordinates Network, 11842, 1

[3] Cenko, S. B., Nugent, P. E., Fox, D. B., Ofek, E. O., \& Kasliwal, M. M. 2011, GRB Coordinates Network, 11827, 1

[4] Kennea, J. A., Romano, P., Krimm, H. A., et al. 2011, The Astronomer's Telegram, 3250, 1

[5] Levan, A. J., Tanvir, N. R., Wiersema, K., \& Perley, D. 2011, GRB Coordinates Network, 11833, 1

[6] Levan, A. J., Tanvir, N. R., Morgan, A., Bloom, J., \& Cenko, S. B. 2011, GRB Coordinates Network, 11846, 1

[7] Levan, A. J., Tanvir, N. R., Cenko, S. B., et al. 2011, Science, 333, 199

[8] van Velzen, S., Körding, E., \& Falcke, H. 2011, MNRAS, 417, L51

[9] Giannios, D., \& Metzger, B. D. 2011, MNRAS, 416, 2102

[10] Bloom, J. S., Butler, N. R., Cenko, S. B., \& Perley, D. A. 2011, GRB Coordinates Network, 11847, 1

[11] Bloom, J. S., Giannios, D., Metzger, B. D., et al. 2011, Science, 333, 203

[12] Burrows, D. N., Kennea, J. A., Ghisellini, G., et al. 2011, Nature, 476, 421

[13] Zauderer, B. A., Berger, E., Soderberg, A. M., et al. 2011, Nature, 476, 425

[14] Berger, E., Zauderer, A., Pooley, G. G., et al. 2012, ApJ, 748, 36

[15] Reis, R. C., Miller, J. M., Reynolds, M. T., et al. 2012, Science, 337, 949

[16] Wiersema, K., van der Horst, A. J., Levan, A. J., et al. 2012, MNRAS, 421, 1942

[17] Sbarufatti, B., Burrows, D.N., Gehrels, N., Kennea, A., 2012 The Astronomer's Telegram, 4398, 1

[18] Cenko, S. B., Krimm, H. A., Horesh, A., et al. 2012, ApJ, 753, 77

[19] Levan, A. J., \& Tanvir, N. R. 2011, GRB Coordinates Network, 11825, 1

[20] Abramowicz, M. A., \& Liu, F. K. 2012, arXiv:1210.2641 